$01,12,13$

\title{
Детерминизм локального атомного упорядочения в монослоях золота в формировании их электронной структуры
}

\author{
() В.Л. Карбовский, А.А. Романский, Л.И. Карбовская, В.В. Стонис \\ Институт металлофизики им. Г.В. Курдюмова НАН Украины, \\ Киев, Украина \\ E-mail: anromansky@gmail.com
}

Поступила в Редакцию 10 ноября 2021 г.

В окончательной редакции 10 ноября 2021 г.

Принята к публикации 11 ноября 2021 г.

\begin{abstract}
В рамках теории функционала плотности были рассчитаны полные и парциальные плотности электронных состояний (ППС) идеального и дефектного монослойного золота толщиной от 1 до 10 монослоев. Внесение вакансий в монослой приводит к смещению главного максимума, а в ряде случаев - расщеплению особенностей на кривой ППС, что приближает конечную форму кривой плотности состояний к таковой для более толстых пластин. Показано, что размещение топологических соседей в образце, вариативность атомных позиций и соответствующих им наборов связей является детерминирующим фактором в формировании кривой ППС пластин благородных металлов. Наибольший вклад здесь вносят преимущественно $d$-состояния. Симметрия плоскости монослоя, так же, как и наличие дефектов упаковки и/или вакансий, существенно влияет на электронные состояния образца и может выступать методом „bulking“ кривой ППС.
\end{abstract}

Ключевые слова: золото, монослой, электронная плотность, дефекты.

DOI: 10.21883/FTT.2022.03.52089.238

\section{1. Введение}

В работе [1] исследовано изменение электронной плотности тонких пленок золота плоскости (111) в зависимости от толщины пленки и концентрации дефектов. Плоскость (111) была выбрана в связи с тем, что экспериментально полученные монослойные пленки золота имеют симметрию плоскости (111) [2-4]. Исследование монослоев других плоскостей может дать крайне важную информацию о закономерностях формирования особенностей электронной структуры как тонких пленок золота, так и объемного металла, а изучение влияния дефектов на этот процесс существенно дополняет систематические данные по формированию общей картины дизайна электронной структуры металлов группы золота.

Несмотря на то что свободное существование монослоев металлов является сомнительным с точки зрения физики твердого тела, некоторые особенности электронной структуры при наличии, например, дефектов, могли бы все же позволить стабильное существование таких структур при определенных условиях. В частности, полученные нами ранее экспериментальные данные [2,3] показывают, что образование монослойных чешуек золота при наличии вакансий и дефектов упаковки все же возможно. Кроме того, важно учесть то, что согласно ряду работ, в частности, $[5,6]$, золото обладает уникальной склонностью к стабильности относительно крупных $2 D$-кластеров, благодаря $5 d-6 s$-гибридизации.

Помимо этого, теоретическое изучение особенностей электронного строения монослоев металлов, в частности - золота, является необходимым шагом для изуче- ния закономерностей послойного роста металлических наноструктур.

На сегодня, изучение свойств и возможностей использования как послойно выращенных наноструктур, так и тонких пленок золота является крайне актуальным, в частности, в электронике [7,8], энергетике [9], плазмонике и создании метаматериалов [10-12].

Механизмы формирования наноструктур золота, как и мелких его кластеров, все еще нуждаются в ряде дополнений, хотя на сегодняшний день уже существует весьма внушительный массив данных. Опираясь на крайне важные современные результаты, в частности - [5,13], эта работа призвана дополнить данные о формировании электронной структуры кластеров и дефектных монослоев золота, а также желанием авторов связать электронно-энергетическое строение монослойных структур золота с исходными физико-химическими данными атомов золота и их топологией на плоскости.

\section{2. Метод исследования}

Полные и парциальные плотности электронных состояний были получены методом тетраэдра [14] с использованием пакета Wien $2 \mathrm{k}[15,16]$. Использовались полнопотенциальный метод (Л)ППВ + ло ((линейного) присоединения плоских волн + локальная орбиталь „the full-potential (L)APW + lo method“) $[17,18]$ и обобщенное градиентное приближение Пердю-БуркеЭрнцерхофа (generalized gradient approximation (GGA) PBE (Perdew, Burke, Ernzerhof)), как наиболее популярный GGA-функционал [19]. Визуализация атомного строения производилась при помощи VESTA [20]. 


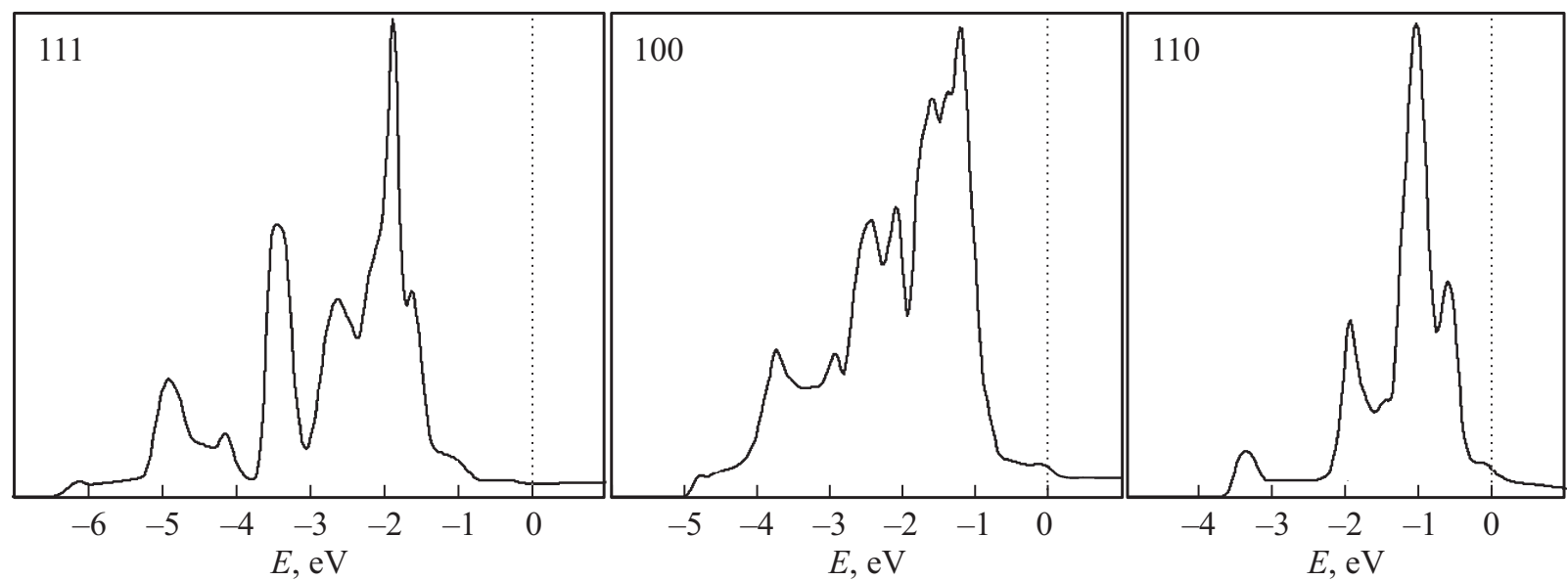

Рис. 1. Рассчитанные TDOS идеальных монослоев плоскостей (111), (110) и (100).
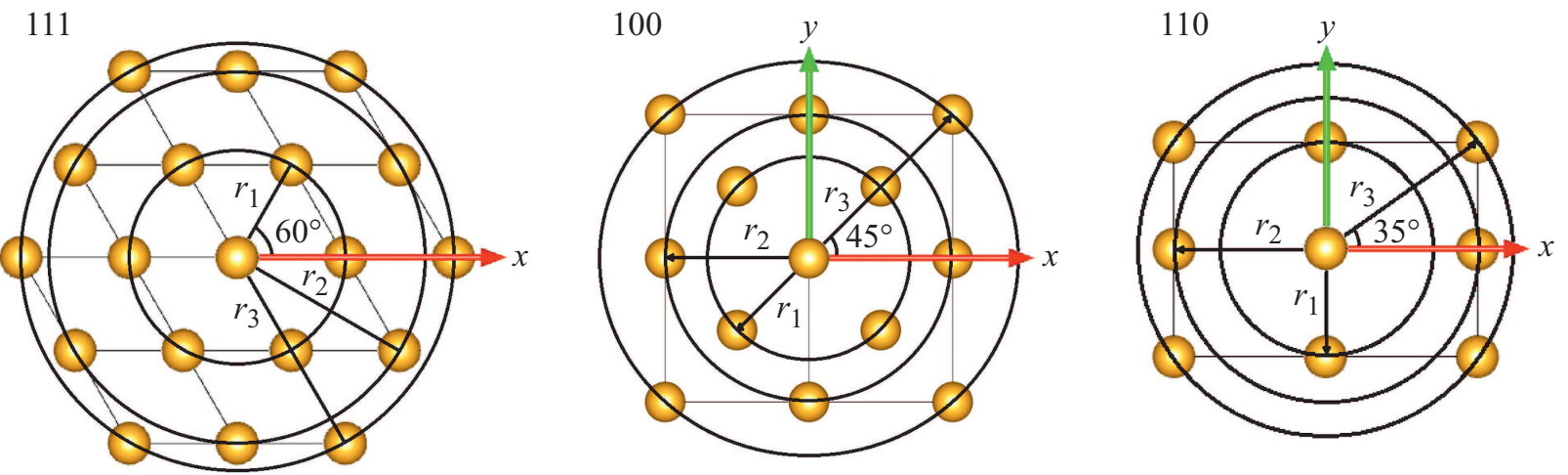

Рис. 2. Первые три координационные сферы и угол $\varphi$ для монослоев плоскостей золота (111), (100) и (110).

\section{3. Результаты и обсуждение}

Полученные кривые полных плотностей электронных состояний (TDOS) монослоев золота приведены на рис. 1 и расположены по степени убывания плотности упаковки атомов (таблица). Кроме того, в этом же ряду наблюдается закономерность уменьшения количества соседей в первой координационной сфере. На расстоянии $2.88 \AA$ для монослоев плоскостей (111), (100)

Энергетическое положение главного максимума на кривой TDOS, межатомные длины связей и координационные числа для различных плоскостей золота

\begin{tabular}{l|c|c|c}
\hline \multicolumn{1}{c|}{ Плоскость золота } & $(111)$ & $(100)$ & $(110)$ \\
\hline Площадь ячейки $\left(\AA^{2}\right)$ & 8.29 & 16.65 & 11.75 \\
Количество атомов на ячейку & 1 & 2 & 1 \\
Положение главного максимума $(\mathrm{eV})$ & $\sim 1.9$ & $\sim 1.3$ & $\sim 1.0$ \\
& $2.88 / 6$ & $2.88 / 4$ & $2.88 / 2$ \\
Длины связей & $4.99 / 6$ & $4.08 / 4$ & $4.08 / 2$ \\
$(\AA) /$ координационное число & $5.77 / 6$ & $5.77 / 4$ & $4.99 / 4$ \\
Атомная плотность (atom per $\left.\mathrm{nm}^{2}\right)$ & 12.06 & 12.01 & 8.51
\end{tabular}

и (110) количество атомов в первой координационной сфере равно 6, 4 и 2 соответственно.

Рассматривая ближайшее окружение атомов во взаимосвязи с особенностями электронного строения важно учитывать не только длину связи, но и конкретное положение атома-соседа в плоскости. Для удобства такое положение будем описывать углом $\varphi$ (рис. 2). На рис. 2 приведены первые три координационные сферы, поскольку, в случае дефектного монослоя (110), изменения в атомном окружении наблюдаются только в третьей координационной сфере.

В работе [1] анализировались изменения кривых TDOS пластин золота плоскости (111) при изменении количества монослоев и наличии вакансий. Было показано, что при определенных концентрациях вакансий в монослое плоскости (111) наблюдается расщепление главных максимумов кривой TDOS, что приближает форму этой кривой к таковой для массивного образца. Каждый атом идеального монослоя золота плоскости (111) имеет в первой координационной сфере 6 ближайших соседей, которые характеризуются разным углом $\varphi$ к оси $x$, что сказывается на различных вкладах компонент $d$-состояний. 
Как видно из рис. 2 плоскости (110) и (100) имеют отличную от (111) вращательную симметрию. Ввиду этого кривые TDOS монослоев золота разных плоскостей имеют существенные различия. Наблюдается существенное сужение валентной полосы $\mathrm{c} \sim 6.5 \mathrm{eV}$ для монослоя плоскости (111) до $\sim 3.5 \mathrm{eV}$ для монослоя плоскости (110). На кривой TDOS монослоя плоскости (110) можно выделить только 3 основных особенности. В отличие от плоскости (110) для плоскости золота (100) характерно наличие атома на отрезке, соединяющем центральный атом и атом третьей координационных сфер (рис. 2, $r_{3}$ ). При этом межатомные расстояния для первой и второй координационных сфер для плоскостей (100) и (110) одинаковы. В случае плоскости (110) углы между линией связи и координатными осями отличаются только в 3-й координационной сфере, однако расстояние в $\sim 5.0 \AA$ является довольно значительным для образования связи и, соответственно, вклад третьей координационной сферы в кривую TDOS предельно мал.

Необходимо отметить, что при другом определении базиса системы координат, углы соответствующих связей все равно сохраняются. При этом изменяются лишь индексы $d$-компонент.

Следовательно, существенные отличия кривых TDOS для монослоев плоскостей (100) и (110) определяются не только наличием дополнительных 2 атомов на расстоянии $4.08 \AA$ для плоскости (100), а главным образом различной локальной симметрией, характерной для данных плоскостей.

При анализе расчетных данных важным критерием является их соответствие экспериментальным данным. Для оценки корректности полученных кривых плотности электронных состояний мы провели их сравнительный анализ с UPS-спектрами (метод ультрафиолетовой фотоэлектронной спектроскопии). В частности, на рис. 3 приведено сопоставление рассчитанной кривой полной плотности состояний пластины, содержащей 3 моноатомных слоя плоскости (110) и спектр валентной полосы золота плоскости (110), полученный методом ультрафиолетовой фотоэлектронной спектроскопии [21].

Известно, что используемый нами квантовомеханический метод расчета электронно-энергетической структуры имеет тенденцию к некоторому энергетическому сжатию получаемых кривых электронных состояний. Как видно, из рис. 3, при незначительном растяжении расчетной кривой можно утверждать о полном соответствии главных особенностей плотности электронных состояний.

Анализ рис. 3 с учетом рис. 4 позволяет утверждать, что главный максимум плотности электронных состояний формируют в основном $d_{x z}$ и $d_{z 2}$ электронные состояния. Пик справа от главного на $\sim 0.5 \mathrm{eV}$ обязан своим происхождением $d_{y z}$ и $d_{x y}$ с незначительной добавкой состояний $d_{x 2-y 2}$, которые также участвуют в формировании и главного максимума. Особенность слева от главного максимума около $2 \mathrm{eV}$ формируется состояниями $d_{y z}$ и $d_{x y}$. Пик на $3.5 \mathrm{eV}$ детерминирован

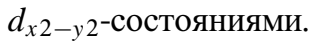

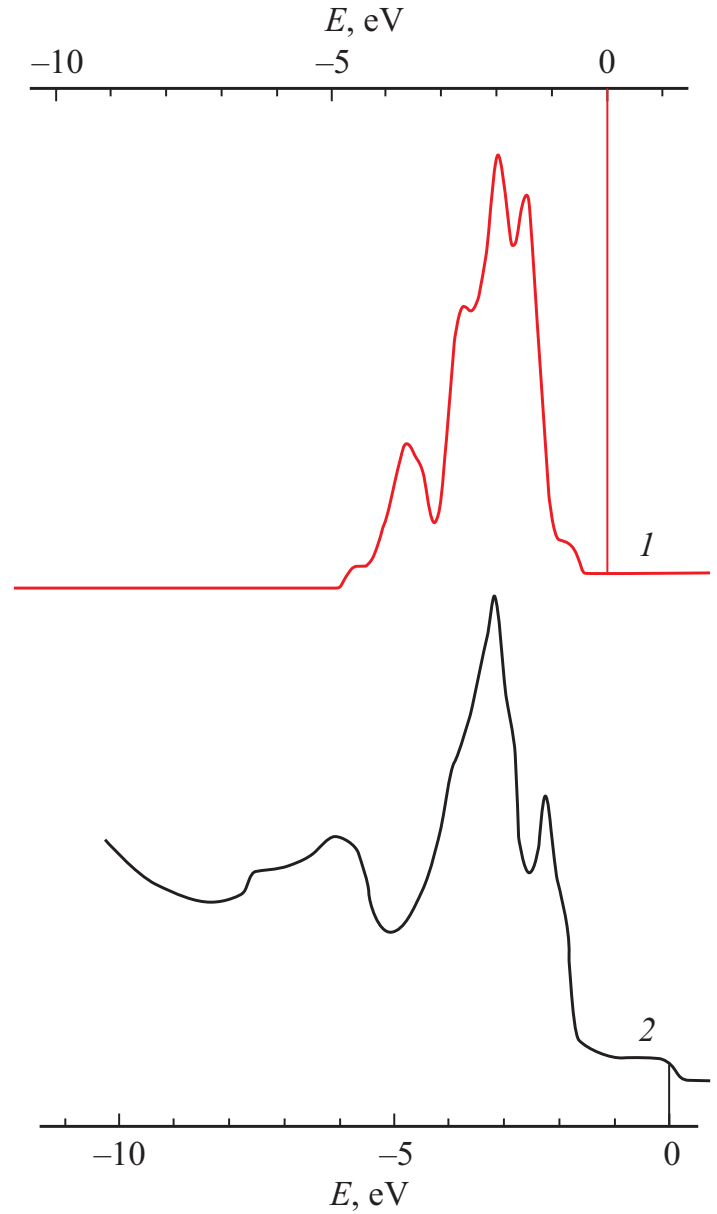

Рис. 3. Рассчитанная кривая TDOS слеба золота плоскости (110) 3 атомных монослоя (1) и UPS-спектр чистой поверхности золота плоскости (110) [21] (2).

Переход к анализу кривой TDOS монослоя плоскости (100) показывает очень богатую тонкую структуру валентной полосы. Атомная плотность в таком монослое в сравнении с плоскостью (110) возрастает почти в 1.4 раза. В первой координационной сфере на том же расстоянии находится уже не 2, а 4 атома.

Исходя из того, что для всех рассматриваемых плоскостей первая координационная сфера атомов находится на одинаковом расстоянии $2.88 \AA$, а изменяется при этом лишь количество атомов в координационной сфере 2,4 и 6 соответственно плоскостям (110), (100) и (111), то очевидно, что первая координационная сфера есть детерминирующей в формировании протяженности и тонкой структуры валентных полос исследуемых структур.

Как было показано ранее [1], в случае плоскости золота (111) вклад в главный максимум плотности состояний вносят преимущественно состояния, связанные с осью $z$. Для (110) такая тенденция сохраняется, но, кроме $z^{2}$ существенный вклад вносит также $x z$-компонента. Точнее сказать, что только $d$-состояния, не связанные (в выбранном базисе) с осью $y$ имеют несколько существенных пиков. 
Таким образом, сложную зонную структуру формируют связи первой координационной сферы. Причем, вариативность углов связей к осям $x$ и $y$ определяет расщепление пиков и формирование более сложной зоны.

Поскольку в исследованных нами ранее $[2,3]$ монослойных чешуйках золота из-за дефектности комбинации связей являются еще более разнообразными, а следовательно - кривая TDOS имеет более развитую тонкую структуру, то исследование дефектных монослоев золота может иметь более прикладной характер, чем расчеты идеальных монослоев. На рис. 5 приведены вклады атомов неэквивалентных позиций в TDOS дефектного монослоя плоскости (110) (суперячейка $2 \times 2$ ).

Наблюдается существенные различия во вкладах в ППС между атомами различных позиций. Пик плотности электронных состояний первого атома образует зону с множеством особенностей, близкую по форме с кривой TDOS, однако значительно менее интенсивную. Этот факт указывает на детерминизм атомов первой позиции в формировании структуры ППС дефектного монослоя золота. Кроме того, интенсивность пиков на кривой ПС атомов первой неэквивалентной позиции меньше, чем на аналогичных кривых атомов второй и третьей неэквивалентной позиций. Скорее всего, такая разница вызвана различиями в атомном окружении. Атом первой

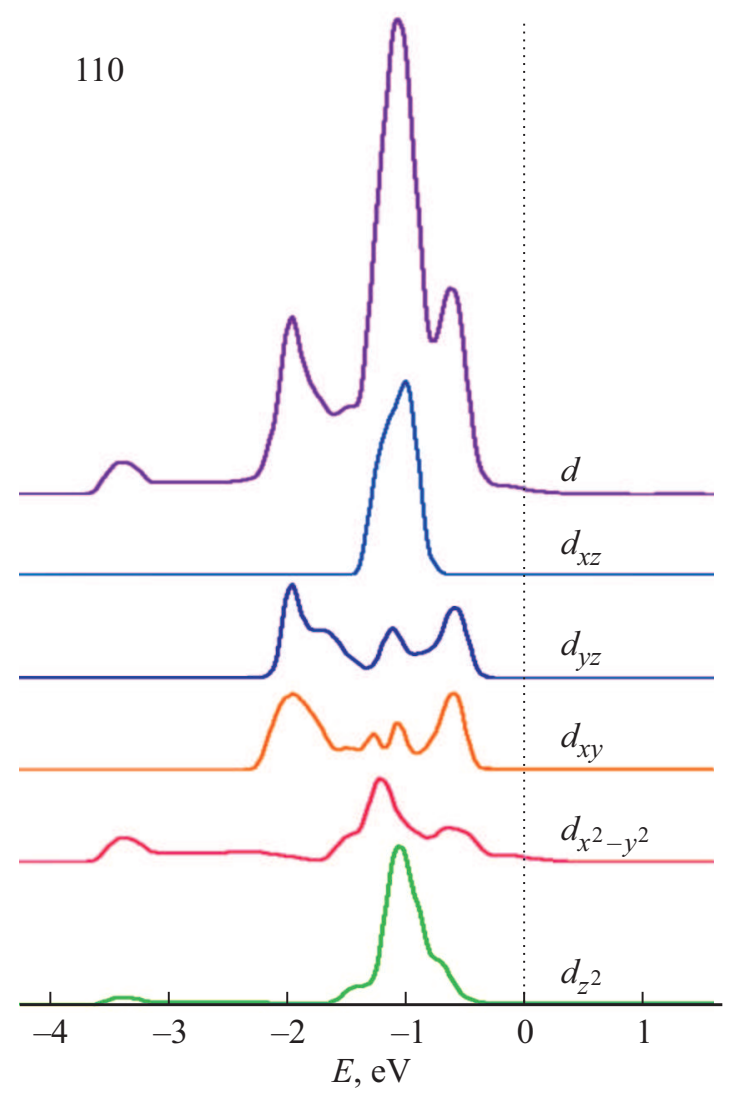

Рис. 4. Рассчитанные парциальные вклады в ППС идеального монослоя плоскости (110).
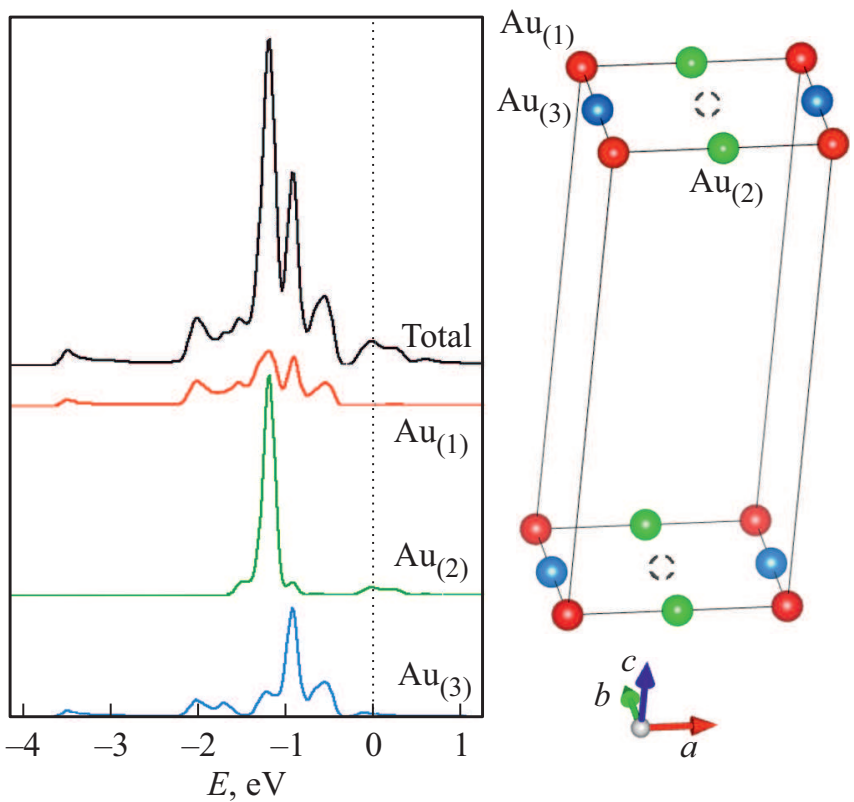

Рис. 5. Вклады атомов неэквивалентных позиций в TDOS дефектного монослоя плоскости (110) (суперячейка $2 \times 2$ ).

неэквивалентной позиции в первой координационной сфере не содержит дефектов. Атомы второй и третьей неэквивалентных позиций соседствуют с вакансией, следовательно, имеет место разрыв связи с ближайшим соседом по одному из направлений.

Для атомов в первой позиции крайне любопытным является тот факт, что изменения атомного окружения в этом случае наблюдаются только в третьей координационной сфере. Таким образом, хотя ближайшие 2 координационные сферы не претерпевают изменений, вклад плотностей состояний атомов первой позиции в общую плотность электронных состояний претерпевает изменения. Следовательно, достаточно отдаленные соседи влияют на плотность состояний.

Кривая $d$-состояний атомов второй позиции имеет очень четкую энергетическую локализацию около $1.2 \mathrm{eV}$, что соответствует положению главного максимума на ППС образца. Анализируя парциальные плотности $d$-состояний для атомов каждой неэквивалентной позиции, можем видеть, что интенсивные пики на кривых плотности состояний для $\mathrm{Au}_{(3)}$ и $\mathrm{Au}_{(2)}$ определяются, в первую очередь, $d$-состояниями, связанными с осями $x$ и $y$.

При наличии вакансий наиболее существенными отличиями между кривыми TDOS идеального и дефектного монослоя является, прежде всего, появление низкоинтенсивной особенности в области около „уровня Ферми“. Длина связи между $\mathrm{Au}_{(1)}$ и $\mathrm{Au}_{(2)}$ по оси $x$ достаточно велика и составляет $4.08 \AA$, в то время как соседи по оси $y$ на расстоянии $\sim 2.88 \AA$ отсутствуют. Ввиду этого атом второй позиции, как видно из рис. 6 , наиболее чувствителен к изменениям.

Для атома первой неэквивалентной позиции наблюдается расщепление главного пика $d_{z 2}$ состояний. При 

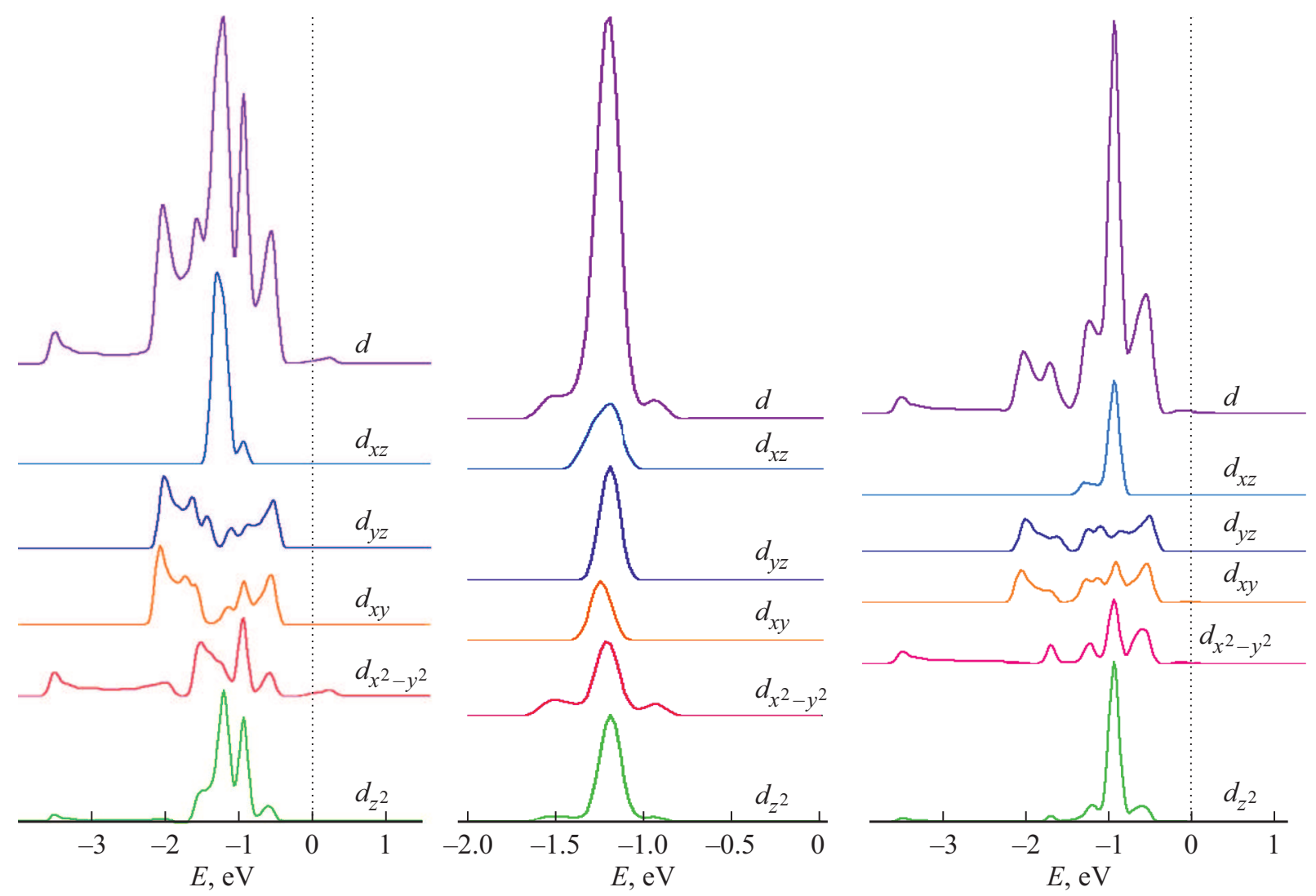

Рис. 6. Рассчитанные парциальные плотности электронных состояний дефектного монослоя плоскости (110).

вакансионной дефектности $2 \times 2 \mathrm{Au}_{(1)}$ не теряет соседей из первой $(\sim 2.88 \AA)$ и второй $(\sim 4.08 \AA)$ координационных сфер, однако, лишается сразу всех соседей из третьей координационной сферы. Учитывая, что это единственное изменение в его окружении, надо полагать, что изменения на кривых TDOS для $\mathrm{Au}_{(1)}$ связаны либо со взаимодействием с атомами третьей координационной сферы, либо с изменением гибридизации различных орбиталей с соседями в первой координационной сфере.

В дефектном монослое плоскости (110) наблюдается незначительное уширение зоны, обусловленое вкладами $d$-состояний, связанными в основном с осью у (в вblбранном базисе). Поскольку в данном случае более короткая связь $\mathrm{Au}_{(1)}-\mathrm{Au}_{(3)}$ лежит именно на оси $y$, можно предположить, что к уширению зоны приводит более сильное взаимодействие с ближайшими соседями.

\section{4. Выводы}

Первая координационная сфера является детерминирующей в формировании тонкой структуры и протяженности валентных полос исследуемых монослойных структур золота. На расщепление пиков кривой TDOS, которое приводит к ее более тонкой структуре, влияют не только длины межатомных связей, но и взаимное расположение атомов.
Показано наличие дальнодействующего влияния атомов третьей координационной сферы на электронное строение монослоев золота. Так для плоскости (110) изменение атомного упорядочения в третьей координационной сфере вследствие внесения вакансии приводит к заметным изменениям на кривой TDOS, что свидетельствует либо о значительной роли атомов третьей координационной сферы, либо о существенном перераспределении взаимодействия $d$-орбиталей различной симметрии близких соседей.

Установлена корреляция между плотностью упаковки, а также количеством соседей в первой координационной сфере и шириной энергетической зоны монослоев золота.

\section{Конфликт интересов}

Авторы заявляют, что у них нет конфликта интересов.

\section{Список литературы}

[1] V.L. Karbivskii, A.A. Romansky, L.I. Karbivska, S.I. Shulyma. Appl. Nanosci (2021). https://doi.org/10.1007/s13204-021-01733-7.

[2] V.L. Karbivskyy, V.V. Vishniak, V.H. Kasiyanenko. J. Adv. Microsc. Res. 6, 278 (2011).

https://doi.org/10.1166/jamr.2011.1083. 
[3] V. Karbivskyy, L. Karbivska, V. Artemyuk. Nanoscale Res. Lett. 11, 69 (2016). https://doi.org/10.1186/s11671-016-1291-2.

[4] L.I. Karbivska, V.L. Karbivskii, A.A. Romansky, O.Y. Kuznetsova, P.O. Teselko, V.A. Artemyuk. Proc. IEEE 39th Int. Conf. on Electronics and Nanotechnology (2019). P. 214. https://doi.org/10.1109/ELNANO.2019.8783939.

[5] H. Häkkinen. Chem. Soc. Rev. 37, 1847 (2008). https://doi.org/10.1039/b717686b.

[6] F. Munoz, A. Varas, J. Rogan, J.A. Valdiviaab, M. Kiwi. Phys. Chem. Chem. Phys. 17, 30492 (2015). https://doi.org/10.1039/c5cp05664k.

[7] C. Liu, Y. Tang, P. Huo, F. Chen. Mater. Lett. 257, 126708 (2019). https://doi.org/10.1016/j.matlet.2019.126708.

[8] G.Y. Jia, Q. Zhang, Z.X. Huang, S.B. Huang, J. Xu. Phys. Chem. Chem. Phys. 19, 27259 (2017). https://doi.org/10.1039/C7CP05260J

[9] A. Kato, M. Suyama, C. Hotehama, H. Kowada, A. Sakuda, A. Hayashi, M. Tatsumisago. J. Electrochem. Soc. 165, A1950 (2018). https://doi.org/10.1149/2.1451809jes.

[10] R. Lemasters, C. Zhang, M. Manjare, W. Zhu, J. Song, S. Urazhdin, H.J. Lezec, A. Agrawal, H. Harutyunyan. ACS Photonics 6, 11, 2600 (2019). https://doi.org/10.1021/acsphotonics.9b00907.

[11] R.A. Maniyara, D. Rodrigo, R. Yu, J. Canet-Ferrer, D.S. Ghosh, R. Yongsunthon, D.E. Baker, A. Rezikyan, F.J. García de Abajo, V. Pruneri. Nature Photon. 13, 328 (2019). https://doi.org/10.1038/s41566-019-0366-X.

[12] O. Guselnikova, P. Postnikov, R. Elashnikov, M. Trusova, Y. Kalachyova, M. Libansky, J. Barek, Z. Kolska, V. Švorčík, O. Lyutakov. Colloids Surf. A 516, 5, 274 (2017). http://dx.doi.org/doi:10.1016/j.colsurfa.2016.12.040.

[13] M.F. Matus, H. Häkkinen. Small. Special Issue: Nanoclusters 17, 27, 2170140 (2021). https://doi.org/10.1002/smll.202170140.

[14] P.E. Blöchl, O. Jepsen, O.K. Andersen. Phys. Rev. B 49, 16223 (1994). https://doi.org/10.1103/PhysRevB.49.16223.

[15] K. Schwarz, P. Blaha, G.K.H. Madsen. Comp. Phys. Commun. 147, 71 (2002). https://doi.org/10.1016/S0010-4655(02)00206-0.

[16] P. Blaha, K. Schwarz, F. Tran, R. Laskowski, G.K.H. Madsen, L.D. Marks. J. Chem. Phys. 152, 074101 (2020). https://doi.org/10.1063/1.5143061.

[17] E. Sjöstedt, L. Nordström, D.J. Singh. Solid State Commun. 114, 15 (2000). https://doi.org/10.1016/S0038-1098(99)00577-3.

[18] G.K.H. Madsen, P. Blaha, K. Schwarz, E. Sjöstedt, L. Nordström. Phys. Rev. B 64, 195134 (2001). https://doi.org/10.1103/PhysRevB.64.195134.

[19] J.P. Perdew, K. Burke, M. Ernzerhof. Phys. Rev. Lett. 77, 3865 (1996). https://doi.org/10.1103/PhysRevLett.77.3865.

[20] K. Momma, F. Izumi. J. Appl. Cryst. 44, 1272 (2011). https://doi.org/10.1107/S0021889811038970.

[21] J.M. Gottfried, K.J. Schmidt, S.L.M. Schroeder, K. Christmann. Surf. Sci. 525, 197 (2003). https://doi.org/10.1016/S0039-6028(02)02559-1.

Редактор Т.Н. Василевская 